\section{Change Over Time in the Pattern of Clinical Response to First-line Biologic Drugs in Patients with Rheumatoid Arthritis: Observational Data in a Real-life Setting}

To the Editor:

In the last 2 decades, the management of rheumatoid arthritis (RA) was dramatically changed by the introduction of the treat-to-target (T2T) approach $^{1,2,3}$ and biologic disease-modifying antirheumatic drugs (bDMARD). The effectiveness of those targeted therapies to achieve remission and low disease activity (LDA) has been demonstrated in randomized controlled trials ${ }^{4}$, progressively increasing the use of bDMARD as a real-life application of the T2T strategy. Nevertheless, in the main international registries, the baseline median disease duration of bDMARD starters has been reported to be significantly high, possibly affecting the overall clinical response to this drug class ${ }^{5,6}$.

To evaluate the real effect of T2T recommendations on RA management with $\mathrm{BDMARD}$, we retrospectively analyzed the baseline characteristics and the 1-year clinical response in a cohort of patients with RA who received a first-line bDMARD in our Rheumatology Unit from September 1999 to December 2015, stratifying the study population according to the period of enrollment (1999-2004, 2005-2009, and 2010-2015) and disease duration (under vs over $2 \mathrm{yrs}$ ), and analyzing data according to an intent-to-treat protocol. Eligible study population was extracted from a local registry approved by the Gaetano Pini Institute Ethics Committee (approval no. 150_2002), including all patients who signed the informed consent for any subsequent retrospective analysis of clinical data. All analyzed clinical information is reported as anonymous aggregate data, excluding any identifiable medical information. Intergroup differences were analyzed by the chi-square test for dichotomous variables and Kruskal-Wallis test for continuous variables. One-year clinical response was defined as the proportion of patients achieving Disease Activity Score at 28 joints (DAS28) remission $(<2.6)$ or LDA $(\geq 2.6 \text { and }<3.2)^{7}$.

The analysis included 725 patients $[82.9 \%$ women, mean age $( \pm$ SD) $53.9 \mathrm{yrs}( \pm 12.5)$, median disease duration (interquartile range) $8.48 \mathrm{yrs}$ (4.34-15.72)] treated with tumor necrosis factor inhibitors $(n=648)$, anakinra $(n=15)$, abatacept $(n=29)$, tocilizumab $(n=27)$, or rituximab $(n=6)$. As reported in Table 1, we observed a significant decrease over time of baseline median disease duration, mean Health Assessment Questionnaire-Disability Index score, and mean DAS28.

Moreover, we found a gradually increased proportion of patients [from $22.9 \%(1999-2004)$ to $37.8 \%(2010-2015), \mathrm{p}=0.0002]$ beginning bDMARD in moderate disease activity (DAS28 $\geq 3.2$ and $<5.1$ ), with a concomitant decrease over time [from $75.5 \%$ (1999-2004) to $58.2 \%$ (2010-2015), p < 0.0001] of bDMARD starters in high disease activity (DAS28 > 5.1). In addition, we observed a progressively increasing number of patients with earlier RA ( $\leq 2 \mathrm{yrs})$ receiving the first bDMARD $(6.9 \%$, 9.8\%, and $14.1 \%$ for $1999-2004,2005-2009$, and 2010-2015 subgroups, respectively). Those data showed the gradual change of biologic-treated population baseline characteristics toward the inclusion of patients with early RA, less disability, and a lower disease activity, demonstrating a better rheumatologists' adherence to T2T strategy. As remarked by Aga, et al in a Norwegian Disease Modifying Antirheumatic Drug Registry cohort analysis, during the last decade clinicians have implemented modern, more aggressive treatment strategies, which will lead to better longterm disease outcomes ${ }^{8}$.

In our study population, $24.8 \%$ and $11.2 \%$ of patients achieved 1 -year remission or LDA, respectively. After stratification by calendar period of enrollment, the 1 -year remission rate was significantly higher $(\mathrm{p}<0.0001)$ in 2010-2015 (35.9\%) and 2005-2009 (30.2\%) subgroups compared with the 1999-2004 subgroup (11.7\%), whereas no difference emerged in comparison of 1-year LDA rate in the 3 evaluated periods $(13.6 \%, 9.8 \%$, and $10.6 \%$, respectively; $\mathrm{p}=0.41$ ). The OR for achieving 1 -year remission was significantly higher in early $(\leq 2 \mathrm{yrs} ; \mathrm{n}=79)$ compared with late $(>2$ yrs; $\mathrm{n}=646)$ patients with RA $(41.8 \%$ vs $22.7 \%$, respectively; OR 2.435 , $95 \%$ CI 1.501-3.950, $\mathrm{p}=0.0005$ ), whereas no differences were observed in 1 -year LDA between the same subgroups $(10.1 \%$ vs $11.3 \%$, respectively; OR $0.8844,95 \%$ CI $0.4092-1.911, \mathrm{p}=0.8519$ ).

Our 16-year, real-life clinical experience clearly demonstrates the increasing tendency of an earlier bDMARD introduction in the disease course, even in patients with moderate disease activity and less severity. Moreover, in the most recently analyzed period (2010-2015), about 50\% of our patients achieved remission/LDA, despite an overall baseline median disease duration of more than 6 years. Even if our study has not been designed and powered for analyzing the rigorousness in the application of the T2T strategy, the described trend may be considered as a reasonable consequence of a better adherence to the European League Against Rheumatism $\mathrm{T} 2 \mathrm{~T}$ recommendations.

ENNIO GIULIO FAVALLI, MD, Department of Rheumatology, Gaetano Pini Institute, Milan, Italy; ANDREA BECCIOLINI, MD, Department of Rheumatology, Gaetano Pini Institute, Milan, Italy; PIER LUIGI MERONI, MD, Department of Clinical Sciences and Community Health, University of Milan and IRCCS Istituto Auxologico Italiano, Milan, Italy. Address correspondence to Dr. E.G. Favalli, Gaetano Pini Institute, Department of Rheumatology, Via Gaetano Pini 9, Milan 20122, Italy. E-mail: ennio.favalli@gmail.com

\section{REFERENCES}

1. Bakker MF, Jacobs JW, Verstappen SM, Bijlsma JW. Tight control in the treatment of rheumatoid arthritis: efficacy and feasibility. Ann Rheum Dis 2007;66 Suppl 3:iii56-60.

2. Grigor C, Capell H, Stirling A, McMahon AD, Lock P, Vallance R, et al. Effect of a treatment strategy of tight control for rheumatoid arthritis (the TICORA study): a single-blind randomised controlled trial. Lancet 2004;364:263-9.

3. Smolen JS, Landewé R, Breedveld FC, Dougados M, Emery P, Gaujoux-Viala C, et al. EULAR recommendations for the

Table 1. Baseline characteristics of study population.

\begin{tabular}{|c|c|c|c|c|}
\hline Characteristics & $1999-2004, n=274$ & $2005-2009, \mathrm{n}=245$ & $2010-2015, \mathrm{n}=206$ & $\mathrm{p}$ \\
\hline Age, yrs, mean (SD) & $54.49(12.66)$ & $53.34(12.17)$ & $53.94(12.73)$ & $0.5629 *$ \\
\hline Disease duration, yrs, median (IQR) & $10.37(5.51-17.09)$ & $7.51(3.79-15.51)$ & $6.64(3.38-15.03)$ & $0.0003 *$ \\
\hline $\mathrm{DAS} 28$, mean $(\mathrm{SD})$ & $5.788(1.154)$ & $5.352(1.158)$ & $5.146(1.333)$ & $<0.0001 *$ \\
\hline HAQ-DI, median (IQR) & $1.75(1.375-2.125)$ & $1.5(1-1.875)$ & $1.125(0.875-1.625)$ & $<0.0001 *$ \\
\hline Methotrexate use, $\%$ & 71.2 & 62 & 59.2 & $0.0003 * *$ \\
\hline
\end{tabular}

* Kruskal-Wallis test. ** Chi-square test. IQR: interquartile range; DAS28: Disease Activity Score at 28 joints; HAQ-DI: Health Assessment Questionnaire-Disability Index. 
management of rheumatoid arthritis with synthetic and biological disease-modifying antirheumatic drugs. Ann Rheum Dis 2010;69:964-75.

4. Nam JL, Ramiro S, Gaujoux-Viala C, Takase K, Leon-Garcia M, Emery P, et al. Efficacy of biological disease-modifying antirheumatic drugs: a systematic literature review informing the 2013 update of the EULAR recommendations for the management of rheumatoid arthritis. Ann Rheum Dis 2014;73:516-28.

5. Neovius M, Arkema EV, Olsson H, Eriksson JK, Kristensen LE, Simard JF, et al; ARTIS Study Group. Drug survival on TNF inhibitors in patients with rheumatoid arthritis comparison of adalimumab, etanercept and infliximab. Ann Rheum Dis 2015;74:354-60.

6. Greenberg JD, Reed G, Decktor D, Harrold L, Furst D, Gibofsky A, et al; CORRONA Investigators. A comparative effectiveness study of adalimumab, etanercept and infliximab in biologically naive and switched rheumatoid arthritis patients: results from the US CORRONA registry. Ann Rheum Dis 2012;71:1134-42.

7. Prevoo ML, van 't Hof MA, Kuper HH, van Leeuwen MA, van de Putte LB, van Riel PL. Modified disease activity scores that include twenty-eight-joint counts. Development and validation in a prospective longitudinal study of patients with rheumatoid arthritis. Arthritis Rheum 1995;38:44-8.

8. Aga AB, Lie E, Uhlig T, Olsen IC, Wierød A, Kalstad S, et al. Time trends in disease activity, response and remission rates in rheumatoid arthritis during the past decade: results from the NOR-DMARD study 2000-2010. Ann Rheum Dis 2015;74:381-8.

J Rheumatol 2017;44:2; doi:10.3899/jrheum.161045 